\title{
Parallel Plate Model for Trabecular Bone Exhibits Volume Fraction-Dependent Bias
}

\author{
J. S. DAY, ${ }^{1}$ M. DING, ${ }^{2}$ A. ODGAARD ${ }^{2}$ D. R. SUMNER, ${ }^{3}$ I. HVID,${ }^{2}$ and H. WEINANS ${ }^{1}$ \\ ${ }^{1}$ Erasmus Orthopaedics Research Lab, Erasmus University, Rotterdam, The Netherlands \\ ${ }^{2}$ Orthopaedic Research Laboratory, Aarhus University Hospital, Aarhus, Denmark \\ ${ }^{3}$ Departments of Anatomy and Orthopedic Surgery, Rush Medical College, Rush-Presbyterian-St. Luke's Medical Center, Chicago, IL, USA
}

\begin{abstract}
Unbiased stereological methods were used in conjunction with microcomputed tomographic (micro-CT) scans of human and animal bone to investigate errors created when the parallel plate model was used to calculate morphometric parameters. Bone samples were obtained from the human proximal tibia, canine distal femur, rat tail, and pig spine and scanned in a micro-CT scanner. Trabecular thickness, trabecular spacing, and trabecular number were calculated using the parallel plate model. Direct thickness, and spacing and connectivity density were calculated using unbiased three-dimensional methods. Both thickness and spacing calculated using the plate model were well correlated to the direct three-dimensional measures $\left(r^{2}=0.77-0.92\right)$. The correlation between trabecular number and connectivity density varied greatly $\left(r^{2}=0.41-0.94\right)$. Whereas trabecular thickness was consistently underestimated using the plate model, trabecular spacing was underestimated at low volume fractions and overestimated at high volume fractions. Use of the plate model resulted in a volume-dependent bias in measures of thickness and spacing $(p<0.001)$. This was a result of the fact that samples of low volume fraction were much more "rod-like" than those of the higher volume fraction. Our findings indicate that the plate model provides biased results, especially when populations with different volume fractions are compared. Therefore, we recommend direct thickness measures when three-dimensional data sets are available. (Bone 27:715-720; 2000) (C) 2000 by Elsevier Science Inc. All rights reserved.
\end{abstract}

Key Words: Trabecular bone; Architecture; Histomorphometry; Microcomputed tomography (micro-CT).

\section{Introduction}

Morphological properties of trabecular bone are often calculated by assuming that the structure consists of a series of parallel plates. ${ }^{24}$ Area fraction and bone perimeter are measured from histological sections. These values are then extrapolated into three dimensions using stereological principles to estimate volume fraction and surface area, which are in turn used to calculate trabecular thickness (Tb.Th), trabecular spacing (Tb.Sp), and

Address for correspondence and reprints: Harrie Weinans, Erasmus Orthopaedic Research Lab, EE1614, Erasmus University Rotterdam, P.O. Box 1738, 3000 DR Rotterdam, The Netherlands. E-mail: weinans@ortho.fgg.eur.nl trabecular number (Tb.N). A summary of the calculations involved is included in the Appendix. Because the area fraction and bone perimeter can be obtained from standard histological sections this method is used widely. However, trabecular bone does not consist of plates, but is in fact a mixture of complex irregular geometries that can at best be approximated as plates and rods. As a result, trabecular thickness and trabecular spacing derived using the plate model are often smaller than when measured directly in three dimensions and may include other model-dependent biases. ${ }^{1,10,20}$

During the past decade new methods have been introduced to obtain three-dimensional data sets of trabecular bone using serial sectioning, ${ }^{21}$ microcomputed tomography (micro-CT), ${ }^{27}$ or magnetic resonance imaging (MRI) ${ }^{18}$ New measures of three-dimensional architecture have accompanied these advances. The direct thickness method provides a model-free measure of trabecular thickness (Tb.Th*) and trabecular spacing (Tb.Sp*). ${ }^{10}$ Using a three-dimensional representation of the trabecular architecture, a series of spheres is fit inside of either the bone or marrow phase. The largest sphere associated with each bone or marrow voxel is found and used to calculate a volume-weighted average thickness or spacing. Histograms can also be created to show the distribution of the parameters, providing further details of the threedimensional (3D) architecture. ${ }^{10}$

Connectivity density can also be calculated directly from three-dimensional data sets using topological principles. Connectivity represents the number of connections that can be severed without separating the network. This is considered to be the number of trabeculae minus one in a topological sense. ${ }^{22}$ Connectivity density is simply the connectivity per unit of volume. The trabecular number, as calculated using the plate model, is simply the inverse of the center-to-center distance between parallel plates and is calculated as the ratio of volume fraction to trabecular thickness. This measure is not necessarily an alternative to connectivity density, but has been used as such.

In addition to the direct thickness method, Hildebrand and Ruegsegger introduced another 3D measure, the structure model index (SMI), which allows objective quantification of how "rodlike" or "plate-like" an object is. ${ }^{11}$ A 3D data set is used to create a smooth model of the bone surface. Using this model, the surface area, first derivative of surface area, and volume are used to calculate the structure model index. An object consisting purely of plates would have a structure model index of 0 and an object consisting purely of rods would have a structure model index of 3. Objects containing a mixture of elements would have intermediate values. In our previous research, we noted that the structure model index has a strong negative correlation with the 
volume fraction. ${ }^{4}$ This relation was also noted in a study of four sites in the human body. ${ }^{9}$ We were concerned that this change of structure type within bone samples of varied volume fraction could influence the histomorphometric parameters obtained using the plate model. The purpose of this work was to use unbiased three-dimensional methods to investigate the errors produced using the plate model with various types of bone samples.

\section{Materials and Methods}

Data were collected from four studies of trabecular architecture in the: (i) human proximal tibia; (ii) canine distal femur; (iii) rat vertebra; and (iv) porcine lumbar spine. Human samples were acquired during a research project investigating the effects of aging in the proximal tibia. ${ }^{3}$ Forty samples were acquired from donors aged 16-85 years. Samples were drilled out from the tibial condyles using a trephine with an inner diameter of 7.5 $\mathrm{mm}$. They were cut $1 \mathrm{~mm}$ beneath the subchondral bone plate and again at the distal end to create a 7.5-mm-long sample. Canine distal femur samples were collected from 38 dogs, aged 14-167 months. All canine samples were D.C. harvested from the femoral intercondylar fossa using a $9.5 \mathrm{~mm}$ trephine, then cut to a length of $10 \mathrm{~mm}$. The first three tail vertebrae were collected from eight rats, aged 6 months. These vertebrae were scanned intact. Eleven L-3 vertebrae were harvested from 6-month-old pigs. The vertebrae endplates and facets were removed and a brick-shaped sample was cut from the trabecular core.

Specimens were scanned using a CT scanner with a maximum resolution of $20 \mu \mathrm{m}$ ( $\mu \mathrm{CT}$ 20; Scanco Medical AG, Zürich, Switzerland). Scanning resulted in cubic voxels with $22,26,11$, and $30 \mu \mathrm{m}$ edge lengths for the human, canine, rat, and pig samples, respectively. After scanning, the data sets were segmented using thresholds that were determined experimentally. ${ }^{5}$ Because the rat vertebrae were scanned intact, the trabecular core was digitally separated from the cortical shell by manually outlining the contour of the boundary in each specimen. A triangularized 3D virtual object was created from the resulting voxel data. This object was used to determine the surface area of the trabecular bone. Trabecular thickness (Tb.Th), trabecular spacing (Tb.Sp), and trabecular number (Tb.N) were determined for all samples using the plate model. Bone volume fraction $(\mathrm{Vv})$, direct trabecular thickness (Tb.Th*) and spacing (Tb.Sp*), connectivity density (CD), and structure model index (SMI) were calculated directly from the $3 \mathrm{D}$ data set.

Unbiased quantification of the connectivity required splitting the data set to avoid edge artifacts. ${ }^{22}$ This method has been described previously. ${ }^{6}$ In short, the dimensions of the data set are reduced by approximately $0.4 \mathrm{~mm}$ to create a smaller inner cylinder and an outer shell. The Euler number of the original set, the smaller cylinder, and the outer shell are calculated, and the Euler number of the interface is defined as the difference between the Euler number of the original object and the sum of the two smaller objects. Half of the connectivity of the interface is added to that of the inner cylinder before calculating the connectivity density of the cylinder. The entire data set was used to calculate all other parameters.

Statistical analyses were performed using a statistical software package (SPSS, SPSS Inc., Chicago, IL). Linear regression models were used to determine how well the model-based parameters were correlated with the direct 3D measurements. To determine whether volume fraction-related changes in the structure type causes a bias in the plate model results, regression models were made for each of the parameters vs. the volume fraction, and the ratio of the model-based/directly
Table 1. Regression of the correlation between the two- and threedimensional parameters

\begin{tabular}{lcccc}
\hline & Dog & Human & Pig & Rat \\
\hline Tb.Th vs. Tb.Th* & & & & \\
$\quad$ Slope & 0.87 & 0.92 & 1.14 & 1.16 \\
Intercept & -0.02 & -0.04 & -0.04 & -0.02 \\
$r^{2}$ & 0.84 & 0.88 & 0.92 & 0.83 \\
$p<$ & 0.001 & 0.001 & 0.001 & 0.001 \\
& & & & \\
Tb.Sp vs. Tb.Sp* & & & & \\
$\quad$ Slope & 1.39 & 1.47 & 1.69 & 0.79 \\
Intercept & -0.22 & -0.22 & -0.36 & 0.02 \\
$r^{2}$ & 0.77 & 0.80 & 0.78 & 0.75 \\
$p<$ & 0.001 & 0.001 & 0.001 & 0.001 \\
& & & & \\
Tb.N vs. CD & & & & \\
$\quad$ Slope & 0.05 & 0.09 & 0.07 & n.a. \\
Intercept & 1.20 & 1.29 & 1.18 & n.a. \\
$r^{2}$ & 0.75 & 0.41 & 0.94 & n.a. \\
$p<$ & 0.001 & 0.001 & 0.001 & n.a. \\
\hline
\end{tabular}

The $p$-value indicates the two-tailed probability that the slope of the regression line is not equal to zero. See text for explanation of bone parameters. n.a., not applicable.

measured parameters vs. volume fraction. Differences in the slopes and intercepts of the two methods were evaluated using the $95 \%$ confidence intervals. Stepwise linear regression was used to determine whether trabecular number correlated with connectivity density after controlling for volume fraction.

\section{Results}

The model-based thickness (Tb.Th) and directly measured thickness (Tb.Th*) were well correlated as were the two measures of trabecular spacing (Tb.Sp and Tb.Sp*). However, the correlation between trabecular number (Tb.N) and connectivity density (CD), while reasonable in the dog and pig, was low in humans. A summary of the regression analyses is displayed in Table $\mathbf{1 .}$

The model-based trabecular thickness was consistently smaller than the directly measured thickness. The slopes of the two were not different when plotted against the volume fraction, and the intercepts were different in the human and dog only. For trabecular spacing, the slopes and intercepts were different for the human and dog when plotted against the volume fraction. For the pig only the intercepts was significantly different, and for the rat there were no significant differences (Figure 1).

To investigate further the difference between the results from the plate model and the direct thickness method, the ratio of the two thickness measures and the ratios of the two spacing measures were plotted against the volume fraction (Figure 2A,B). There was a significant relation in all cases (Table 2). This trend did not always exist for the ratio of trabecular number to connectivity density (Figure 2C).

Although the one-dimensional (1D) trabecular number is not directly comparable to the $3 \mathrm{D}$ connectivity density there was a moderate correlation between these parameters (Table 1). Stepwise linear regression indicated that prediction of the connectivity density was significantly increased when the trabecular number was added to a model of volume fraction alone $(p<0.05)$.

As was the case in our previous studies, there was a strong inverse relation between structure model index and volume fraction (Figure 3). 


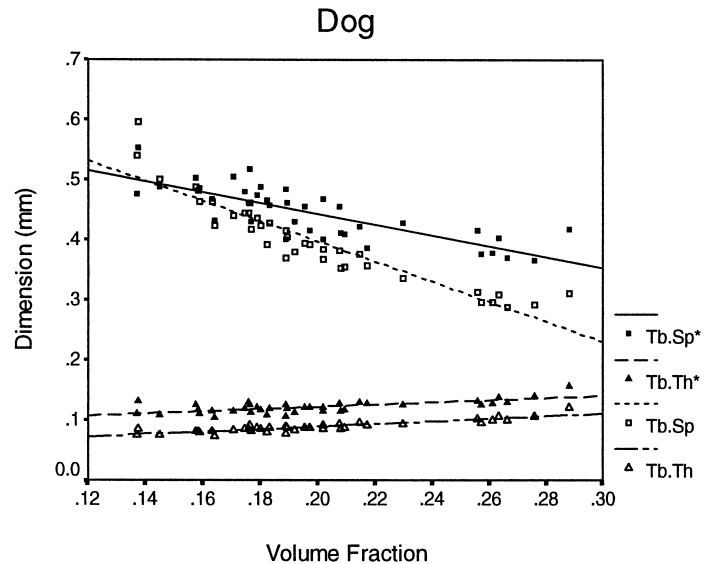

Pig

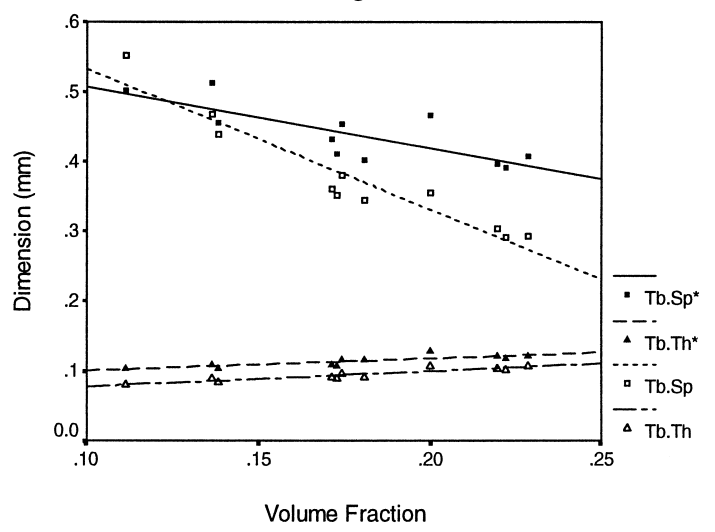

Plate model and volume fraction-dependent bias

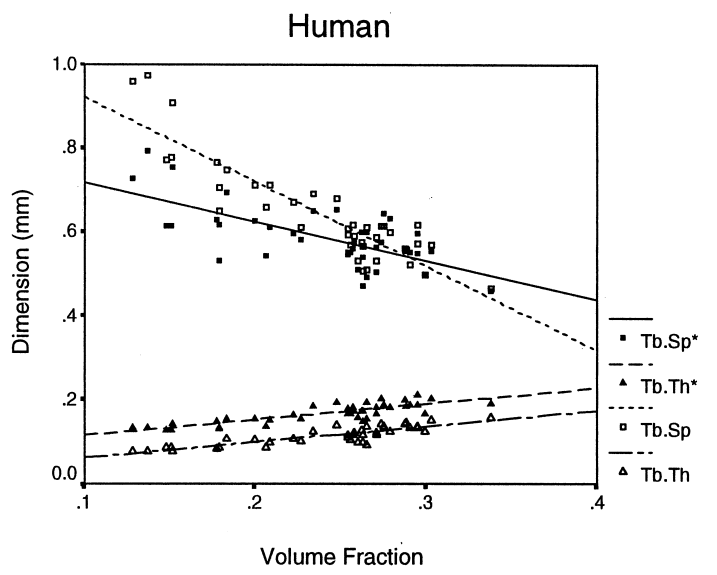

Rat

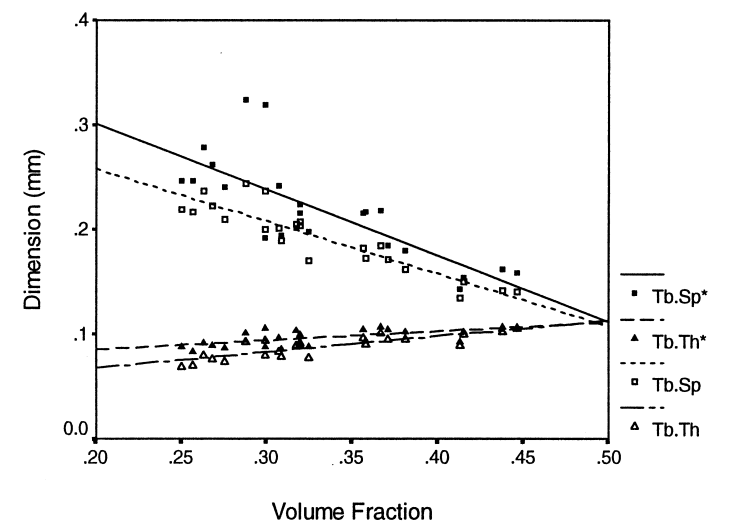

Figure 1. Two-dimensional (2D) and three-dimensional (3D) parameters plotted against varying volume fraction. Trabecular thickness was consistently underestimated by the plate model. For trabecular spacing the plate model overestimated spacing at low volume fractions but underestimated as the volume fraction increased.

\section{Discussion}

In this work we have shown that there is a volume fractiondependent bias in the morphometric parameters determined using the plate model. A similar bias has previously been noted in a two-dimensional (2D) comparison of directly measured and model-based thickness. ${ }^{1}$ As illustrated by the nearly parallel regression lines in Figure 1, there was a consistent difference of up to $40 \mu \mathrm{m}$ between the model-based and directly measured trabecular thickness, independent of volume fraction. Hildebrand et al. ${ }^{9}$ reported that the plate model underestimates trabecular thickness. This finding has been repeated in our study. However, the magnitude of the increase in thickness relative to increased volume fraction was similar for both the 2D and 3D methods. This could be problematic if changes in thickness measured using the plate model are interpreted as percentages of the original thickness. In this case, use of the plate model would result in exaggerated relative changes in thickness. This is clearly illustrated in Figure 2. The relation between model-based and directly measured trabecular spacing was not as simple as for thickness. Plate model spacing was neither consistently underestimated nor overestimated (Figure 1). The differences in the slopes of the regression lines resulted in volume fraction-dependent bias, which is illustrated in Figure 2.

To use model-based methods to determine morphological parameters, assumptions must be made about the structure of the material. Although the plate model can be modified for use with rod-like or plate-like materials, the model type cannot be used effectively when materials are composed of an unknown proportion of plates and rods. It is the need for a fixed model type that leads to bias in the model. By using the structure model index to measure how "rod-like" or "plate-like" the samples used in this study were, the mechanism responsible for bias in the plate model could be demonstrated (Figure 3). The structure model index had a strong inverse relation to volume fraction, that is, as the volume fraction increased, the structure model index decreased, indicating that the material was more plate-like. The inverse relation between structure model index and volume fraction may have been due to the perforation of trabecular plates during resorption. As platelike trabeculae are perforated, they become more rod-like and present a greater surface area. Thus, their trabecular thickness is systematically underestimated using the plate model. The converse is true when using a rod-based model. As volume fraction increases there are more plate-like structures with thicknesses that are systematically overestimated. The strong relation between structure model index and volume fraction has been noted in previous 3D studies of trabecular architecture. $^{4,9}$

Connectivity is a measure of the number of branches in the trabecular network that may be cut without separating the structure. As a topological property, connectivity density cannot be measured using 2D data. However, the trabecular 

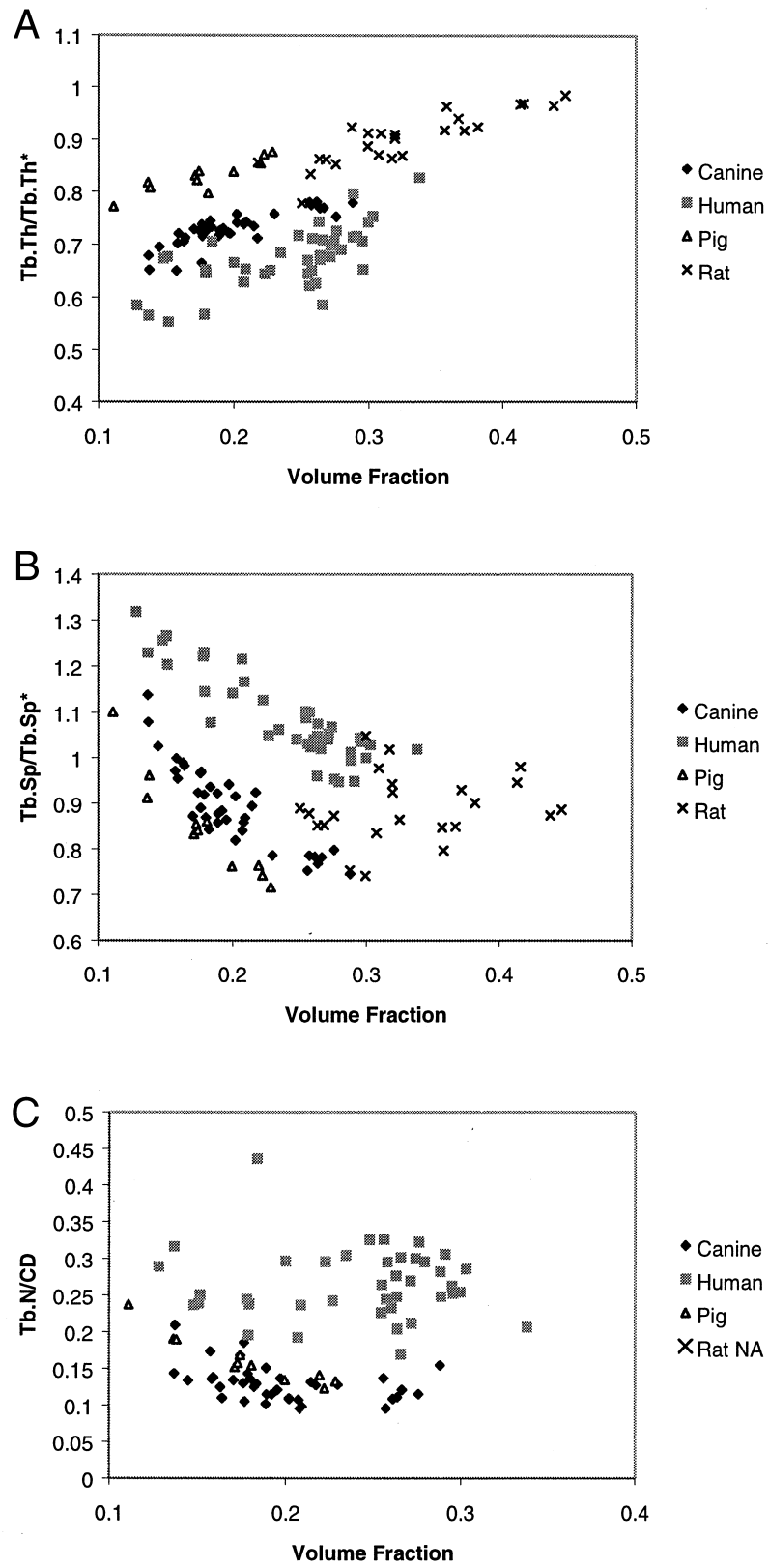

Figure 2. Illustration of volume fraction-dependent bias in trabecular thickness, spacing, and number. The ratios between the model-based and directly measured parameters were plotted against the volume fraction.

number and connectivity density, or connectivity per unit volume, were significantly correlated. This has been demonstrated previously. ${ }^{22}$ There was no significant volume fraction-related bias in the ratio of the two connectivity measures. Stepwise regression analysis indicated that the trabecular number was a better predictor of connectivity density than volume fraction alone, particularly for canine bone. This indicates that the trabecular number does have some utility in estimating the connectivity density. However, the use of connectivity as a measure of bone quality is a much debated subject. $^{12,15,16}$

Although unbiased 3D methods have been presented in the literature, model-based methods are still commonly used to
Table 2. Regression of volume fraction-dependent bias

\begin{tabular}{lcccc}
\hline & Dog & Human & Pig & Rat \\
\hline Tb.Th/Tb.Th* vs. Vv & & & & \\
$\quad$ Slope & 0.67 & 0.75 & 0.76 & 0.70 \\
Intercept & 0.60 & 0.49 & 0.70 & 0.67 \\
$r^{2}$ & 0.68 & 0.48 & 0.79 & 0.75 \\
$p<$ & 0.001 & 0.001 & 0.001 & 0.001 \\
& & & & \\
Tb.Sp/Tb.Sp* vs. Vv & & & & \\
$\quad$ Slope & -1.98 & -1.59 & -0.28 & -0.11 \\
Intercept & 1.29 & 1.46 & 1.34 & 0.93 \\
$r^{2}$ & 0.76 & 0.78 & 0.91 & 0.03 \\
$p<$ & 0.001 & 0.001 & 0.001 & n.s. \\
& & & & \\
Tb.N/CD vs. Vv & & & & \\
$\quad$ Slope & -0.24 & -0.06 & -0.81 & n.a. \\
Intercept & 0.18 & 0.28 & 0.30 & n.a. \\
$r^{2}$ & 0.15 & 0.00 & 0.89 & n.a. \\
$p<$ & 0.05 & n.s. & 0.001 & n.a. \\
\hline
\end{tabular}

The $p$-value indicates the two-tailed probability that the slope of the regression line is not equal to zero. See text for explanation of bone parameters. n.a., not applicable; n.s., not significant.

examine 3D data sets..$^{2,14,29,30}$ Where 3D data are available only direct unbiased methods should be used to measure histomorphometric parameters.

In this work, we have chosen to use the direct thickness measure ${ }^{10}$ as the "gold standard." This method calculates a volume-weighted average thickness from a 3D data set and assigns heavier weighting to larger trabeculae. It is possible that thin trabecula form the "weak link" in terms of the mechanical properties of the structure. Another possibility for weighting includes skeletonizing the trabecular structure and calculating the thickness at regular intervals along the branches. This produces a length-weighted measure of trabecular thickness and has been used in a two-dimensional study of trabecular bone. 7

The results presented in this work are from a small number of selected locations and do not represent the full range of volume fractions seen in the body. In the samples investigated there was a strong negative correlation between volume fraction and the structure model index. This indicates that bone becomes more "rod-like" at lower volume fractions and that

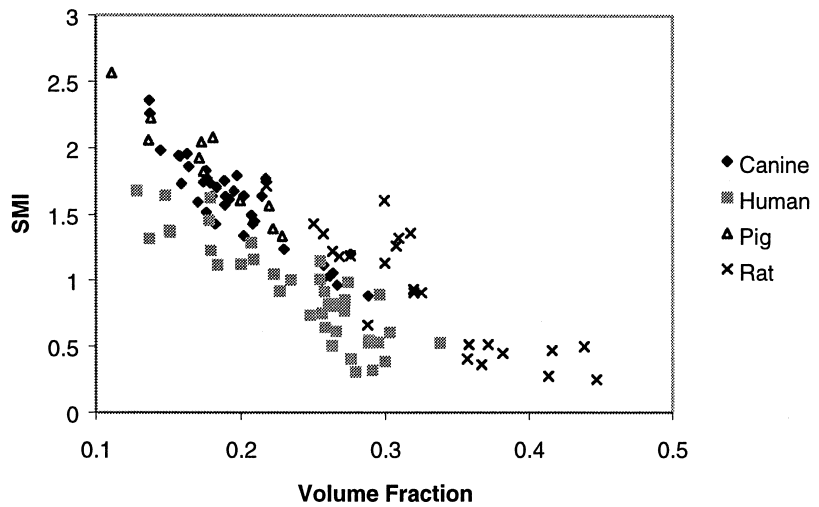

Figure 3. Structure model index was plotted against the volume fraction. A material composed of ideal rods would have a structure model index of 3 , whereas a structure composed only of plates would have a structure model index of 0 . There was a strong negative correlation between structure model index and volume fraction. 
this trend is quite predictable. If true, one may be tempted to make a generalized correction to the plate model such that it can be adjusted to more accurately represent the direct thickness. One may try to justify this correction by citing the high correlation between the direct and model-based methods. However, our results varied with the species investigated and may have been further affected by the scanning resolution. In addition, it must be stressed that this relation may not be valid for diseased bone. Thus, a generalized correction to the plate model is not appropriate for the study of pathologic states or drug treatments.

The plate model is commonly used in studies where changes in trabecular morphology are examined in the presence of changes in volume fraction. This is true for studies of aging, ${ }^{19,24,25,28}$ osteoporosis, ${ }^{17,26}$ osteoarthritis, ${ }^{13}$ the effect of electromagnetic fields, ${ }^{8}$ and many others. Because of the convenience and relatively low expense of two-dimensional histomorphometry its use will probably continue in the future. When conventional 2D histomorphometry is used to measure trabecular morphology care must be taken when interpreting the results. It must be remembered at all times that the only parameters that are truly measured using this method are the bone perimeter and bone area. A reported change in trabecular thickness may be simply the result of a change in volume fraction. Conversely, it may be the result of a change in structure type of the specimen. The only way to determine whether there are pathological changes in bone structure that are different from the normal change associated with a change in volume fraction would be to plot the surface/volume ratio against the volume fraction and determine whether there is a difference in the regression lines.

The use of model-based methods requires that adjustments be made for both the structure type and anisotropy of the material (see Appendix). In anisotropic structures, the orientation of the cutting plane can markedly influence the measured parameters. Object dimensions vary greatly depending on whether they are viewed from a direction perpendicular or parallel to the principal axis of anisotropy. ${ }^{31}$ Thickness, as calculated using the plate (or rod) model is the ratio of perimeter to area and nothing more. Correcting for anisotropy, as well as the choice of a plate model, rod model, or a combination of the two, requires a priori knowledge of the structure of the sample. Thus, the conversion to trabecular thickness simply provides a conceptual tool that is useful for the interpretation of the data. The calculations of trabecular number and trabecular spacing are extensions of this conceptual tool, fraught with the same limitations. Thickness will be underestimated using the plate model and there will be volume fraction-dependent biases, especially in the estimation of trabecular spacing. The trabecular number provides only a rough estimate of the true connectivity, but is free of volume fraction-dependent bias. It is not possible to predict the effects of disease or new drug treatments upon the model assumptions and therefore we do not see the possibility for generalized correction of these model-based parameters.

Acknowledgments: Funding for this work was from NIH Grants AR14685 and AR42862 and Danish Health Research Council Grant 9601833_lpa. The authors thank Aivars Berzins for preparing canine femoral samples, Hans Gregersen and Jingbo Zhao for contributing rat vertebrae, and Wolf Drescher for supplying pig vertebrae. The research of Harrie Weinans was made possible by The Netherlands Royal Academy of Arts and Sciences.

\section{References}

1. Birkenhager-Frenkel, D. H., Courpron, P., Hupscher, E. A., Clermonts, E., Coutinho, M. F., Schmitz, P. I., and Meunier, P. J. Age-related changes in cancellous bone structure. A two-dimensional study in the transiliac and iliac crest biopsy sites. Bone Miner 4:197-216; 1988.

2. Ciarelli, T. E., Fyhrie, D. P., Schaffler, M. B., and Goldstein, S. A. Variations in three-dimensional cancellous bone architecture of the proximal femur in female hip fractures and in controls. J Bone Miner Res 15:32-40; 2000.

3. Ding, M., Dalstra, M., Danielson, C. C., Kabel, J., Hvid, I., and Linde, F. Age variations in the properties of human tibial trabecular bone. J Bone Jt Surg [Br] 79:99-100; 1997.

4. Ding, M., Odgaard, A., Danielson, C. C., and Hvid, I. Young's modulus of cancellous bone predicted by 3-D structural properties and densities. In press.

5. Ding, M., Odgaard, A., and Hvid, I. Accuracy of cancellous bone volume fraction measured by micro-CT scanning. J Biomech 32:323-326; 1999.

6. Ding, M., Odgaard, A., Linde, F., and Hvid, I. Age variations in the microstructure of human tibial cancellous bone. J Orthop Res. In press.

7. Garrahan, N. J., Mellish, R. W., Vedi, S., and Compston, J. E. Measurement of mean trabecular plate thickness by a new computerized method. Bone 8:227-230; 1987.

8. Gonzalez-Riola, J., Pamies, J. A., Hernandez, E. R. Revilla, M., Seco, C., Villa, L. F., and Rico, H. Influence of electromagnetic fields on bone mass and growth in developing rats: A morphometric, densitometric, and histomorphometric study. Calcif Tissue Int 60:533-537; 1997.

9. Hildebrand, T., Laib, A., Muller, R., Dequeker, J., and Ruegsegger, P. Direct three-dimensional morphometric analysis of human cancellous bone: Microstructural data from spine, femur, iliac crest, and calcaneus. J Bone Miner Res 14:1167-1174; 1999.

10. Hildebrand, T. and Ruegsegger, P. A new method for the model independent assessment of thickness in three-dimensional images. J Microsc 185:67-75; 1997.

11. Hildebrand, T. and Ruegsegger, P. Quantification of bone microarchitecture with the structure model index. Comp Meth Biomech Biomed Eng 1:15-23; 1997.

12. Kabel, J., Odgaard, A., van Rietbergen, B., and Huiskes, R. Connectivity and the elastic properties of cancellous bone. Bone 23:115-120; 1999

13. Kamibayashi, L., Wyss, U. P., Cooke, T. D., and Zee, B. Trabecular microstructure in the medial condyle of the proximal tibia of patients with knee osteoarthritis. Bone 17:27-35; 1995.

14. Kapadia, R. D., Stroup, G. B., Badger, A. M., Koller, B., Levin, J. M., Coatney, R. W., Dodds, R. A., Liang, X., Lark, M. W., and Gowen, M. Applications of micro-CT and MR microscopy to study pre-clinical models of osteoporosis and osteoarthritis. Technol Health Care 6:361-372; 1998

15. Kinney, J. H. Connectivity and the elastic properties of cancellous bone [letter]. Bone 25:741-747; 1999.

16. Kinney, J. H. and Ladd, A. J. The relationship between three-dimensional connectivity and the elastic properties of trabecular bone. J Bone Miner Res 13:839-840; 1998.

17. Kleerekoper, M., Villanueva, A. R., Stanciu, J., Rao, D. S., and Parfitt, A. M. The role of three-dimensional trabecular microstructure in the pathogenesis of vertebral compression fractures. Calcif Tissue Int 37:594-597; 1985.

18. Majumdar, S. A review of the magnetic resonance (MR) imaging of trabecular bone microarchitecture: Contribution to the prediction of biomechanical properties and fracture prevalence. Technol Health Care 6:321327; 1998.

19. Mellish, R. W., Garrahan, N. J., and Compston, J. E. Age-related changes in trabecular width and spacing in human iliac crest biopsies. Bone Miner 6:331-338; 1989.

20. Odgaard, A. Three-dimensional methods for quantification of cancellous bone architecture. Bone 20:315-328; 1997.

21. Odgaard, A., Andersen, K., Melsen, F., and Gundersen, H. J. A direct method for fast three-dimensional serial reconstruction. J Microsc 159:335$342 ; 1990$

22. Odgaard, A. and Gundersen, H. J. Quantification of connectivity in cancellous bone, with special emphasis on 3-D reconstructions. Bone 14:173$182 ; 1993$.

23. Parfitt, A. M., Drezner, M. K., Glorieux, F. H., Kanis, J. A., Malluche, H., Meunier, P. J., Ott, S. M., and Recker, R. R. Bone histomorphometry: Standardization of nomenclature, symbols, and units. Report of the ASBMR Histomorphometry Nomenclature Committee. J Bone Miner Res 2:595-610; 1987.

24. Parfitt, A. M., Mathews, C. H., Villanueva, A. R., Kleerekoper, M., Frame, 
B., and Rao, D. S. Relationships between surface, volume, and thickness of iliac trabecular bone in aging and in osteoporosis. Implications for the microanatomic and cellular mechanisms of bone loss. J Clin Invest 72 : 1396-1409; 1983.

25. Parisien, M. V., McMahon, D., Pushparaj, N., and Dempster, D. W. Trabecular architecture in iliac crest bone biopsies: Infra-individual variability in structural parameters and changes with age. Bone 9:289-295; 1988.

26. Poodenphant, J., Nielsen, V. A., Riis, B. J., Gotfredsen, A., and Christiansen, C. Bone mass, bone structure and vertebral fractures in osteoporotic patients. Bone 8:127-130; 1987.

27. Ruegsegger, P., Koller, B., and Muller, R. A microtomographic system for the nondestructive evaluation of bone architecture. Calcif Tissue Int 58:24-29; 1996.

28. Snyder, B. D., Piazza, S., Edwards, W. T., and Hayes, W. C. Role of trabecular morphology in the etiology of age-related vertebral fractures. Calcif Tissue Int 53(Suppl.):S14-S22; 1993.

29. Uchiyama, T., Tanizawa, T., Muramatsu, H., Endo, N., Takahashi, H. E., and Hara, T. A morphometric comparison of trabecular structure of human ilium between microcomputed tomography and conventional histomorphometry. Calcif Tissue Int 61:493-498; 1997.

30. Uchiyama, T., Tanizawa, T., Muramatsu, H., Endo, N., Takahashi, H. E., and Hara, T. Three-dimensional microstructural analysis of human trabecular bone in relation to its mechanical properties. Bone 25:487-491; 1999

31. Whitehouse, W. J. Errors in area measurement in thick sections, with special reference to trabecular bone. J Microsc 107:183-187; 1976

\section{Appendix}

Formulas for morphometric parameters ${ }^{23}$

$\mathrm{BV} / \mathrm{TV}=\mathrm{B} \cdot \mathrm{Ar} / \mathrm{TA}$

$\mathrm{BS} / \mathrm{BV}=\mathrm{B} . \mathrm{Pm} / \mathrm{B} . \mathrm{Ar} * 4 / \pi($ see note $)$

Plate model

$\mathrm{Tb} . \mathrm{Th}=2 /(\mathrm{BS} / \mathrm{BV})$

$\mathrm{Tb} . \mathrm{N}=(\mathrm{BV} / \mathrm{TV}) / \mathrm{Tb} . \mathrm{Th}$

$\mathrm{Tb} . \mathrm{Sp}=(1 / \mathrm{Tb} . \mathrm{N})-\mathrm{Tb} . \mathrm{Th}$

Rod model

Tb.Dm $=4 /(\mathrm{BS} / \mathrm{BV})$

Tb.N $=\left(4 / \pi * \mathrm{BV} / \mathrm{TV}^{\wedge} 0.5 / \mathrm{Tb} . \mathrm{Dm}\right.$

$\left.\mathrm{Tb} . \mathrm{Sp}=\mathrm{Tb} . \mathrm{Dm} *((\pi / 4 * \mathrm{TV}) / \mathrm{BV})^{\wedge} 0.5-1\right)$

Note. The value $4 / \pi$ (1.27) can be modified to correct for anisotropy in specimens. A value of 1.2 has been determined experimentally for use in the human ilium.
Date Received: April 24, 2000

Date Revised: July 19, 2000

Date Accepted: July 22, 2000 